\title{
OPEN Transcapillary transport of water, small solutes and proteins during hemodialysis
}

\author{
Leszek Pstras $^{1 凶}$, Jacek Waniewski ${ }^{1}$ \& Bengt Lindholm²
}

The semipermeable capillary walls not only enable the removal of excess body water and solutes during hemodialysis (HD) but also provide an essential mechanism for maintaining cardiovascular homeostasis. Here, we investigated transcapillary transport processes on the whole-body level using the three-pore model of the capillary endothelium with large, small and ultrasmall pores. The transcapillary transport and cardiovascular response to a 4-h hemodialysis (HD) with 2 L ultrafiltration were analyzed by simulations in a virtual patient using the three-pore model of the capillary wall integrated in the whole-body compartmental model of the cardiovascular system with baroreflex mechanisms. The three-pore model revealed substantial changes during HD in the magnitude and direction of transcapillary water flows through small and ultrasmall pores and associated changes in the transcapillary convective transport of proteins and small solutes. The fraction of total capillary hydraulic conductivity attributed to ultrasmall pores was found to play an important role in the transcapillary water transport during HD thus influencing the cardiovascular response to HD. The presented model provides a novel computational framework for a detailed analysis of microvascular exchange during HD and as such may contribute to a better understanding of dialysis-induced changes in blood volume and blood pressure.

Microvascular exchange of water and solutes between the blood and tissues plays a key role in the function and homeostasis of the human body. The transport properties of the exchange vessels vary between different organs and tissues depending on the type of capillaries (continuous, fenestrated or sinusoidal) and may change in some pathological conditions ${ }^{1}$. The magnitude and direction of transcapillary water and solute transport does also change with time depending on the physiological circumstances. On the whole-body level, under steady-state conditions, there is a net filtration of water and solutes (including proteins) from the blood to the tissues, which is compensated by the equivalent lymphatic absorption of interstitial fluid and its transport back to the circulation in the form of lymph ${ }^{2,3}$. During hemodialysis (HD), when relatively large quantities of water and solutes are removed from the patient's body over a few hours, the normal transcapillary filtration becomes progressively reduced and eventually reverses into absorption of fluid from the interstitial space in order to compensate for the reduced blood volume to maintain cardiovascular stability (vascular refilling mechanism ${ }^{4-7}$ ). Given that intradialytic hypotension remains the major complication in $\mathrm{HD}^{8,9}$, study of transcapillary shifts of water and solutes, in particular proteins, may provide important insights to the understanding of this highly undesired phenomenon and could potentially contribute to improvements in the safety and effectiveness of HD therapy.

Over time, there have been multiple approaches to mathematically model the microvascular exchange processes ${ }^{10,11}$. For the continuous capillaries, which are dominant in the human body (e.g. in skeletal muscles, skin, fat tissue, heart and lungs), the currently accepted and widely used concept is the three-pore model $(3 \mathrm{PM})^{12,13}$, which attempts to reflect the structural properties of continuous capillaries by identifying three separate channels for solute and/or water transport (see Fig. 1): (a) large pores (LP) with radius $\sim 250 \AA$ representing large inter-endothelial gaps or junctions that allow an almost unobstructed passage of most plasma macromolecules; (b) small pores (SP) with radius $\sim 45 \AA$ representing clefts between the endothelial cells allowing the passage of all ions and small solutes and a limited passage of small proteins, such as albumin; and (c) ultrasmall pores (UP) with radius $\sim 2 \AA$ representing transcellular aquaporins that allow only water to pass ${ }^{12,13}$.

Given that different pores leak different amounts of proteins, depending on their reflection (or sieving) coefficients, the colloid osmotic pressure gradient develops to a different extent across different types of pores (the same, albeit much smaller effect applies to the osmotic pressure gradient exerted by the small solutes). Hence,

${ }^{1}$ Nalecz Institute of Biocybernetics and Biomedical Engineering, Polish Academy of Sciences, Ks. Trojdena 4, 02-109 Warsaw, Poland. 'Division of Renal Medicine and Baxter Novum, Department of Clinical Science, Intervention and Technology, Karolinska Institute, Stockholm, Sweden. ${ }^{\circledR}$ email: leszek.pstras@ibib.waw.pl 
ultrasmall transcellular pores (UP)

$$
\mathrm{R} \approx 2 \AA
$$

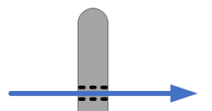

small pores (SP)

$R \approx 45 \AA$

large pores (LP)

$\mathrm{R} \approx 250 \AA$

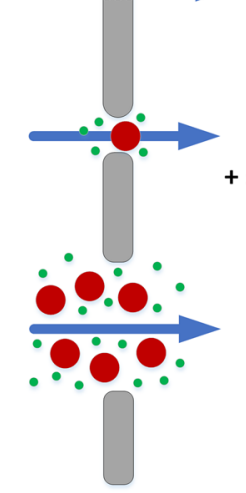

Transport of water only ( $\Delta \pi$ dominates)

Transport of water $(\Delta \mathrm{P}-\Delta \pi)$

+ ions \& small molecules

+ some (limited) macromolecules/proteins

Transport of water ( $\Delta \mathrm{P}$ dominates)

+ ions \& small molecules

+ macromolecules/proteins

Figure 1. The three-pore model of the transcapillary transport of water, small solutes and macromolecules. The transport of water is driven by the hydraulic-hydrostatic pressure difference between capillary blood and interstitial fluid $(\Delta \mathrm{P})$ counteracted by the osmotic pressure difference between plasma and interstitial fluid $(\Delta \pi)$.

despite the same hydraulic-hydrostatic pressure difference acting across all types of pores, the actual rate of fluid filtration through different pores may not reflect their relative hydraulic capacity ${ }^{14}$. For instance, a very low oncotic pressure difference develops across the highly-permeable large pores, and hence a relatively large filtration should be expected to occur via this transport channel, despite its relatively low area and low hydraulic conductivity. Under non-steady conditions, such as during HD, the magnitude and direction of water and solute flow through different pores may vary substantially.

The aim of this study was to investigate the microvascular fluid shifts on the whole-body level using the $3 \mathrm{PM}$ and to assess the impact of relative hydraulic capacities of individual types of capillary pores on the overall cardiovascular response to HD.

\section{Materials and methods}

Model overview. The simulations shown is this study are based on our earlier lumped-parameter compartmental model of the human cardiovascular system with baroregulation ${ }^{15,16}$ integrated with the model of the whole-body water and solute transport designed for modeling of the cardiovascular response to $\mathrm{HD}^{17}$. The proposed model is a non-pulsatile model describing the two-phase blood flow (plasma with suspended erythrocytes) across nine cardiovascular compartments and three extracorporeal compartments, as shown in Fig. 2. The cardiovascular model is equipped with four baroreflex mechanisms controlling peripheral resistance, heart rate, heart contractility and venous unstressed volume. The model includes the aggregated whole-body transport of water and solutes across the capillary wall, tissue cell membrane and erythrocyte membrane as well as via the lymphatic system. The solutes considered in the model include ions $\left(\mathrm{Na}^{+}, \mathrm{K}^{+}, \mathrm{Cl}^{-}, \mathrm{HCO}_{3}^{-}\right.$, and other cations and anions treated collectively), small solutes (urea and creatinine) and proteins (albumin and all other proteins treated collectively as globulins).

The model describes changes in pressures, volumes and composition of all considered fluids in individual compartments (blood, plasma, interstitial fluid, intracellular fluid). The model is based on the set of ordinary differential equations and was implemented in MATLAB (The MathWorks Inc.). For a full description of the model and all model parameters, see our earlier work ${ }^{17}$.

Transcapillary transport model. In terms of transcapillary transport, our original mode ${ }^{17}$ uses the classic homogeneous membrane model, which for the purpose of this study was replaced by the 3PM. The rate of fluid exchange between the capillary compartment and the interstitial compartment was calculated separately for each type of pores depending on the imbalance between the Starling forces acting across the pores: the hydraulic capillary blood pressure, the hydrostatic interstitial pressure and the osmotic (mainly oncotic) pressures exerted by all solutes on both sides of the capillary wall (see Eq. 1 in the Supplementary Information S1).

The radii of large, small and ultrasmall pores were assumed at $250 \AA, 45 \AA$ and $2 \AA$, respectively ${ }^{18}$. The total capillary hydraulic conductivity (LpS) was assumed at $4.5 \mathrm{~mL} / \mathrm{min} / \mathrm{mmHg}^{4,19}$, of which $5 \%, 85 \%$, and $10 \%$ were attributed to LP, SP, and UP, respectively. Based on the assumed pore radii ${ }^{18}$ and hydrodynamic (Stokes-Einstein) solute radii, the reflection coefficients and permeability-surface products (PS) were calculated for all solutes for small and large pores, as shown in Table 1. For all equations describing the 3PM, see the Supplementary Information S1 available online.

To better describe the contribution of small ions to the osmotic pressure gradient across the capillary pores during HD, contrary to our previous work, in which we used the same osmotic coefficient for all ions (0.93), in this study we used separate osmotic coefficients as follows: $\mathrm{Na} 0.94, \mathrm{~K} 0.92, \mathrm{Cl} 0.93, \mathrm{HCO}_{3} 0.96$, other cations $\left(\mathrm{Ca}^{2+}, \mathrm{Mg}^{2+}\right)$ 0.80, other anions $\left(\mathrm{SO}^{2-}, \mathrm{H} 2 \mathrm{PO}^{-}, \mathrm{HPO}^{2-}, \mathrm{PO}^{3-}\right) 0.70^{20}$. 


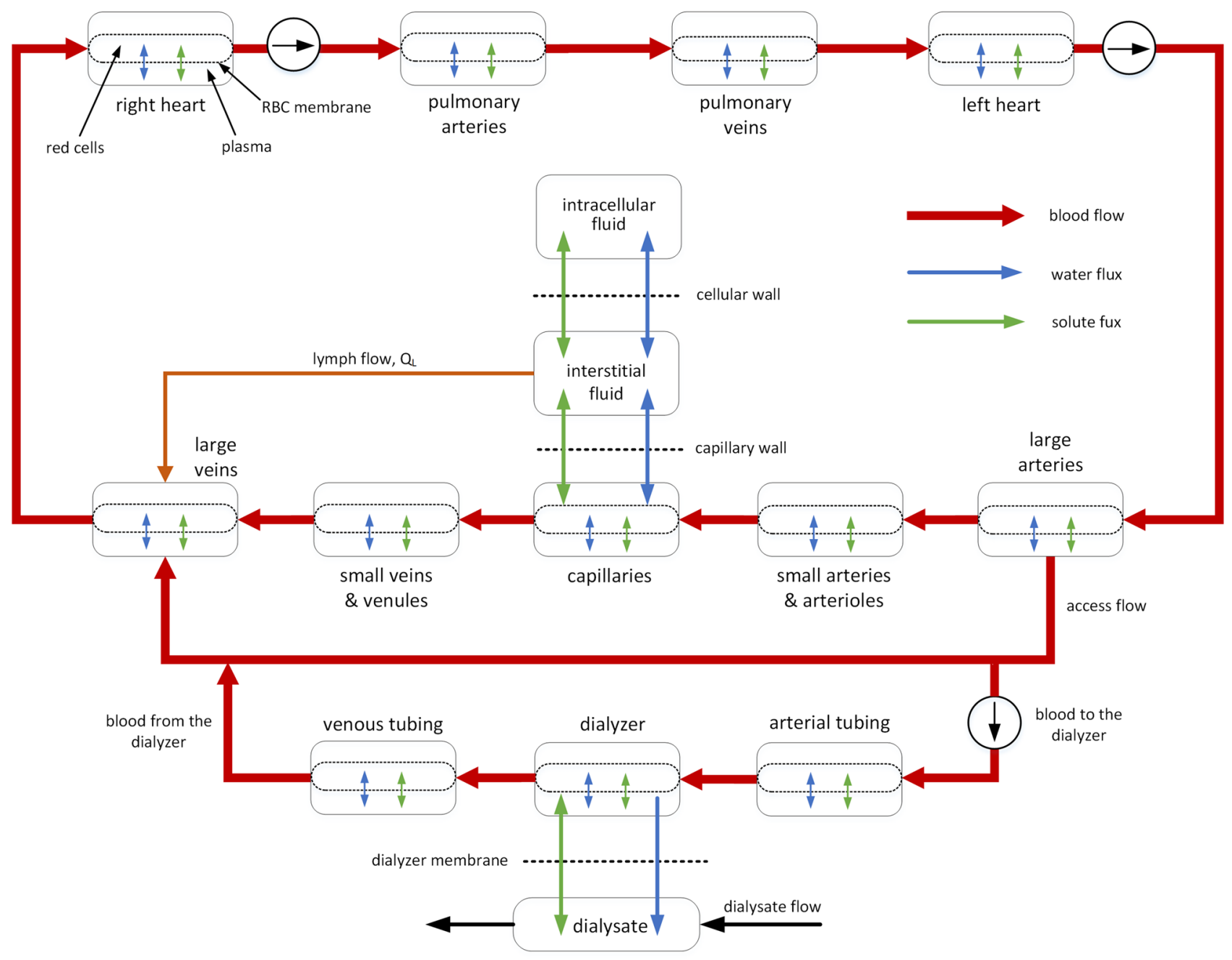

Figure 2. Overview of the integrated model of the cardiovascular system, extracorporeal dialyzer circuit and the whole-body water and solute exchange (based on Pstras and Waniewski ${ }^{17}$ ).

\begin{tabular}{|l|l|l|l|l|l|}
\hline & $\mathbf{r}_{\mathbf{s}}[\AA]$ & $\boldsymbol{\sigma}_{\text {LP }}$ & $\boldsymbol{\sigma}_{\text {SP }}$ & $\mathbf{P S}_{\text {LP }}[\mathbf{m L} / \mathbf{s}]$ & $\mathbf{P S}_{\text {SP }}[\mathrm{mL} / \mathbf{s}]$ \\
\hline Sodium $\left(\mathrm{Na}^{+}\right)$ & 1.8 & 0.00024 & 0.0072 & 0.44 & 200.78 \\
\hline Potassium $\left(\mathrm{K}^{+}\right)$ & 1.3 & 0.00013 & 0.0038 & 0.61 & 291.71 \\
\hline Chloride $\left(\mathrm{Cl}^{-}\right)$ & 1.2 & 0.00011 & 0.0032 & 0.67 & 319.05 \\
\hline Bicarbonate $\left(\mathrm{HCO}_{3}^{-}\right)$ & 2.2 & 0.00036 & 0.0107 & 0.36 & 157.97 \\
\hline Other cations $\left(\mathrm{C}^{2+}\right)$ & 3.1 & 0.00071 & 0.0209 & 0.25 & 102.44 \\
\hline Urea (U) & 1.8 & 0.00024 & 0.0072 & 0.44 & 200.78 \\
\hline Creatinine (Cr) & 2.6 & 0.00050 & 0.0148 & 0.30 & 128.46 \\
\hline Albumin (Alb) & 35.5 & 0.08260 & 0.9442 & 0.012 & 0.0082 \\
\hline Globulins (Glob) & $69.9^{\mathrm{a}}$ & 0.27320 & 1.0000 & 0.003 & 0.0000 \\
\hline
\end{tabular}

Table 1. Assumed hydrodynamic (Stokes-Einstein) solute radii $\left(\mathrm{r}_{\mathrm{s}}\right)^{21-28}$ and calculated reflection coefficients $(\sigma)$ and permeability-surface products (PS) for large pores (LP) and small pores (SP). For ultrasmall pores $\sigma_{\mathrm{UP}}=1$ and $\mathrm{PS}_{\mathrm{UP}}=0$ for all solutes. ${ }^{\mathrm{a}}$ Average hydrodynamic radius of non-albumin plasma proteins calculated from data on individual globulin fractions and fibrinogen ${ }^{28-30}$.

Initial conditions. The model was defined for the steady-state conditions of a pre-dialysis virtual patient with several parameters modified with respect to a reference healthy man based on the literature data ${ }^{17}$ (see Table 2). In terms of fluid overload, knowing that dialysis patients accumulate the excess fluid almost exclusively in the extracellular fluid ${ }^{2}$, for simplicity we assumed that all excess fluid $(2 \mathrm{~L})$ goes to the interstitial fluid, and hence we simply increased the initial volume of the interstitial compartment with respect to the normal man, leaving all vascular compartments unchanged (thus assuming normal blood pressures in all compartments) ${ }^{17}$. 


\begin{tabular}{|c|c|c|}
\hline & Normal & pre-dialysis \\
\hline \multicolumn{3}{|l|}{ Plasma solutes $^{\mathrm{a}}$} \\
\hline Sodium $\left(\mathrm{Na}^{+}\right)(\mathrm{mmol} / \mathrm{L})$ & 140 & $138^{\mathrm{b}}$ \\
\hline Potassium $\left(\mathrm{K}^{+}\right)(\mathrm{mmol} / \mathrm{L})$ & 4.3 & 5 \\
\hline Chloride $\left(\mathrm{Cl}^{-}\right)(\mathrm{mmol} / \mathrm{L})$ & 103 & 103 \\
\hline Bicarbonate $\left(\mathrm{HCO}^{-}\right)(\mathrm{mmol} / \mathrm{L})$ & 26 & 22 \\
\hline Other cations $\left(\mathrm{Ca}^{2+}, \mathrm{Mg}^{2+}\right)(\mathrm{mmol} / \mathrm{L})$ & 2 & 2 \\
\hline Urea $(\mathrm{mmol} / \mathrm{L})$ & 6 & 27 \\
\hline Creatinine $(\mathrm{mmol} / \mathrm{L})$ & 0.1 & 1 \\
\hline Albumin $(\mathrm{g} / \mathrm{dL})$ & 4.1 & 3.75 \\
\hline Total protein $(\mathrm{g} / \mathrm{dL})$ & 7.0 & 6.5 \\
\hline Plasma water fraction $(-)$ & 0.94 & 0.945 \\
\hline Hematocrit (\%) & 47 & 35 \\
\hline Cardiac output (L/min) & 5.25 & 6.2 \\
\hline Fistula blood flow ( $\mathrm{mL} / \mathrm{min})$ & - & 950 \\
\hline
\end{tabular}

Table 2. Assumed patient characteristics ${ }^{17}$. ${ }^{a}$ For small ions, the concentrations listed above are assumed to be the concentrations of free ions that exert osmotic pressure, thus excluding the fractions of ions bounded to proteins or paired/complexed with other ions (those fractions are likely of similar order of magnitude on both sides of capillary walls). ${ }^{\mathrm{b}}$ An average pre-dialysis plasma sodium level typically seen in hemodialysis patients ${ }^{31,32}$.

The composition of the interstitial fluid (i.e. its water fraction and the concentrations of all solutes), the mean capillary blood pressure, and the Gibbs-Donnan coefficient for monovalent cations ( $\alpha$ ) were all found numerically to obtain initial steady-state conditions of the whole system based on the assumed composition of plasma (see Table 2 ) and the normal lymphatic absorption $(8 \mathrm{~L} / \text { day })^{17}$.

Hemodialysis procedure. We analyzed a standard 4-h HD session including the pre-dialysis procedure of filling the extracorporeal circuit with the patient's blood, with the priming fluid (normal saline) infused to the patient, and a couple of minutes of idle circulation before the start of the actual dialysis ${ }^{17}$. The assumed volume of the extracorporeal circuit was $220 \mathrm{~mL}$ and the dialyzer ultrafiltration was set to $9.25 \mathrm{~mL} / \mathrm{min}$ to remove all excess fluid including the priming volume $(2.22 \mathrm{~L})$. The assumed composition of the dialysis fluid was as follows: $\mathrm{Na}^{+} 140 \mathrm{mmol} / \mathrm{L}, \mathrm{K}^{+} 2 \mathrm{mmol} / \mathrm{L}, \mathrm{Cl}^{-} 108 \mathrm{mmol} / \mathrm{L}, \mathrm{HCO}^{-} 34 \mathrm{mmol} / \mathrm{L}$, other cations $\left(\mathrm{Mg}^{2+}, \mathrm{Ca}^{2+}\right) 2 \mathrm{mmol} / \mathrm{L}$. For all parameters of the HD procedure, including the clearances/dialysances of individual solutes, see our earlier work $^{17}$.

Sensitivity analysis. Given that this study focuses ultimately on the cardiovascular response to HD, the mean arterial pressure in the large arteries (MAP) at the end of a 4-h HD session was treated as the main model output for which the sensitivity to small changes in the values of individual model parameters was investigated. The dimensionless relative local sensitivity of the model output y (MAP) to each studied parameter, $\theta_{\mathrm{k}}$, was calculated as follows ${ }^{33,34}$ :

$$
S_{k}=\left.\frac{\partial y}{\partial \theta_{k}} \cdot \frac{\theta_{k}}{y}\right|_{\theta_{k}=\theta_{k, 0}}, \quad \theta_{k}, y \neq 0
$$

where the derivative was computed using the central difference approximation with the parameter $\theta_{\mathrm{k}}$ increased and decreased by $0.01 \%$.

The primary sensitivity analysis was restricted to the parameters related to the 3PM of the transcapillary fluid and solute transport (27 parameters in total): whole-body hydraulic conductivity of capillary walls (LpS), fractions of LpS contributed by the $\mathrm{i}$-th type of pores $\left(\alpha_{\mathrm{i}}\right)$, solute hydrodynamic radii $\left(\mathrm{r}_{\mathrm{s}}\right)$, pore radii $\left(\mathrm{r}_{\mathrm{i}}\right)$, osmotic coefficients of small ions $\left(\varphi_{s}\right)$, molecular weight of proteins $\left(\mathrm{MW}_{\mathrm{p}}\right)$, density of proteins $\left(\rho_{\mathrm{p}}\right)$, electric charge of albumin, globulins (non-albumin proteins) and "other anions". Note that some of these parameters are used directly in the model equations, whereas some others, such as solute and pore radii, are used to calculate the equation parameters, such as solute reflection coefficients $\left(\sigma_{\mathrm{s}}\right)$ and solute permeability-surface products $\left(\mathrm{PS}_{\mathrm{s}}\right)$ see the Supplementary Information S1.

Additionally, we performed a separate sensitivity analysis with respect to all other model parameters (137 in total), including those describing the cardiovascular and extravascular compartments, baroreflex mechanisms, cardiac function, red blood cells, and tissue cells, as well as parameters related to the dialysis session, such as ultrafiltration rate, dialyzer blood flow rate, volume of the dialyzer and dialyzer tubing, composition of the dialysis fluid, and solute clearances/dialysances (the values of all these parameters and the model equations that use them may be found in our previous work ${ }^{17}$ ). 


\begin{tabular}{|l|r|l|l|r|}
\hline & LP & SP & UP & Total \\
\hline Fluid filtration, $\mathrm{mL} / \mathrm{h}$ & 178.2 & 196.3 & 6.1 & 380.6 \\
\hline Water filtration, $\mathrm{mL} / \mathrm{h}$ & 171.8 & 196.1 & 6.1 & 374.0 \\
\hline Albumin diffusion, $\mathrm{mol} / \mathrm{h}$ & 11.84 & 8.08 & - & 19.92 \\
\hline Globulins diffusion, $\mathrm{mol} / \mathrm{h}$ & 1.06 & - & - & 1.06 \\
\hline Albumin convection, $\mathrm{mol} / \mathrm{h}$ & 78.07 & 4.54 & - & 82.61 \\
\hline Globulins convection, $\mathrm{mol} / \mathrm{h}$ & 19.90 & - & - & 19.90 \\
\hline Albumin escape rate, \%/h & 4.69 & 0.66 & - & 5.35 \\
\hline Globulins escape rate, \%/h & 3.68 & - & - & 3.68 \\
\hline Mean capillary pressure, mmHg & 15.43 & & \\
\hline Interstitial fluid pressure, $\mathrm{mmHg}$ & 0.12 & & \\
\hline Interstitial-to-plasma albumin ratio & 0.49 & & \\
\hline Interstitial-to-plasma globulins ratio & 0.34 &
\end{tabular}

Table 3. Transcapillary water and protein transport (from blood to tissues) across large (LP), small (SP) and ultrasmall (UP) pores under pre-dialysis steady-state conditions.

\begin{tabular}{|l|c|c|c|c|c|c|}
\hline & \multicolumn{2}{|l}{$\boldsymbol{\alpha}_{\text {UP }}$} \\
\cline { 2 - 7 } & $\mathbf{0 . 0 5}$ & $\mathbf{0 . 1 0}$ & $\mathbf{0 . 2 0}$ & $\mathbf{0 . 3 0}$ & $\mathbf{0 . 4 0}$ & $\mathbf{0 . 5 0}$ \\
\hline Filtration through LP, \% & 46.7 & 46.8 & 47.2 & 47.5 & 48.0 & 48.6 \\
\hline Filtration through SP, \% & 52.6 & 51.6 & 49.4 & 47.1 & 44.6 & 42.2 \\
\hline Filtration through UP, \% & 0.8 & 1.6 & 3.5 & 5.4 & 7.4 & 9.2 \\
\hline Albumin escape rate, \%/h & 5.37 & 5.35 & 5.33 & 5.31 & 5.29 & 5.28 \\
\hline Globulins escape rate, \%/h & 3.66 & 3.68 & 3.70 & 3.73 & 3.77 & 3.81 \\
\hline Mean capillary pressure, mmHg & 15.38 & 15.43 & 15.52 & 15.63 & 15.76 & 15.92 \\
\hline Interstitial-to-plasma albumin ratio & 0.50 & 0.49 & 0.49 & 0.49 & 0.49 & 0.49 \\
\hline Interstitial-to-plasma globulins ratio & 0.34 & 0.34 & 0.34 & 0.34 & 0.35 & 0.35 \\
\hline
\end{tabular}

Table 4. Transcapillary water and protein transport (from blood to tissues) under pre-dialysis steady-state conditions with different fractions of total hydraulic conductivity (LpS) contributed by ultrasmall pores $\left(\alpha_{U P}\right)$ and the contribution of large pores $\left(\alpha_{\mathrm{LP}}\right)$ kept at 0.05 .

\begin{tabular}{|l|c|c|c|c|c|c|}
\hline & \multicolumn{2}{|l}{$\boldsymbol{\alpha}_{\text {LP }}$} \\
\cline { 2 - 7 } & $\mathbf{0 . 0 3}$ & $\mathbf{0 . 0 4}$ & $\mathbf{0 . 0 5}$ & $\mathbf{0 . 0 6}$ & $\mathbf{0 . 0 7}$ & $\mathbf{0 . 0 8}$ \\
\hline Filtration through LP, \% & 32.5 & 40.2 & 46.8 & 52.4 & 57.3 & 61.4 \\
\hline Filtration through SP, \% & 65.2 & 57.8 & 51.6 & 46.2 & 41.5 & 37.6 \\
\hline Filtration through UP, \% & 2.2 & 1.9 & 1.6 & 1.4 & 1.2 & 1.0 \\
\hline Albumin escape rate, \%/h & 4.06 & 4.76 & 5.35 & 5.86 & 6.30 & 6.69 \\
\hline Globulins escape rate, \%/h & 2.55 & 3.16 & 3.68 & 4.12 & 4.50 & 4.82 \\
\hline Mean capillary pressure, mmHg & 17.80 & 16.55 & 15.43 & 14.43 & 13.53 & 12.73 \\
\hline Interstitial-to-plasma albumin ratio & 0.37 & 0.44 & 0.49 & 0.54 & 0.58 & 0.62 \\
\hline Interstitial-to-plasma globulins ratio & 0.24 & 0.29 & 0.34 & 0.38 & 0.41 & 0.45 \\
\hline
\end{tabular}

Table 5. Transcapillary water and protein transport (from blood to tissues) under pre-dialysis steady-state conditions with different fractions of total hydraulic conductivity (LpS) contributed by large pores $\left(\alpha_{\mathrm{LP}}\right)$ and the contribution of ultrasmall pores $\left(\alpha_{U P}\right)$ kept at 0.10 .

\section{Results}

Pre-dialysis steady state. The characteristics of the whole-body transcapillary water and protein transport across different types of pores under pre-dialysis steady-state conditions are shown in Table 3 . We also compared the pre-dialysis steady-state conditions obtained for different fractions of total hydraulic capillary conductivity contributed by ultrasmall pores $\left(\alpha_{U P}\right)$, keeping the large pore fraction $\left(\alpha_{L P}\right)$ unchanged at 0.05 (see Table 4). We then performed a similar analysis of the impact of large pore fraction, keeping the ultrasmall pore fraction at 0.10 (see Table 5). 

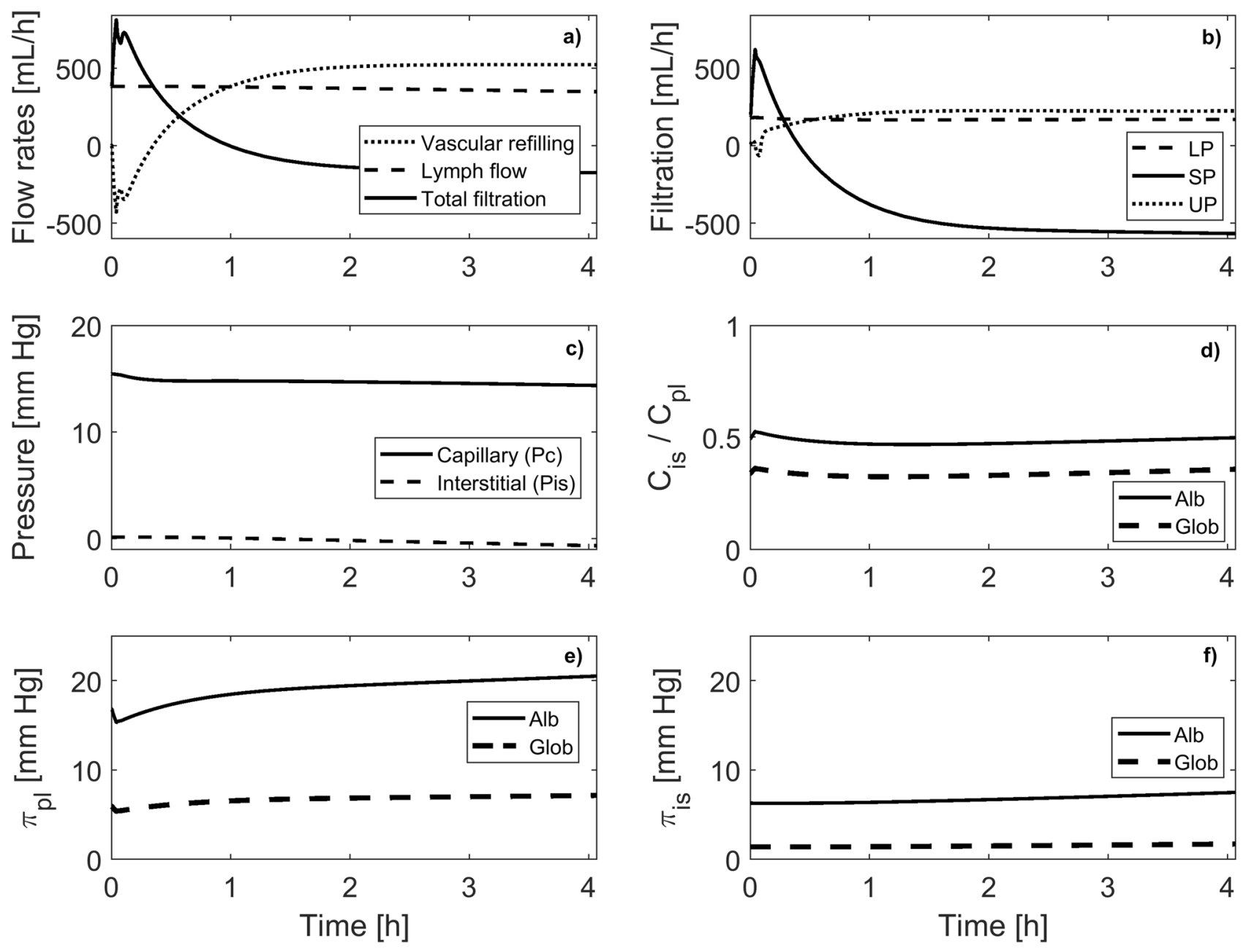

Figure 3. Model-simulated changes in transcapillary transport variables during hemodialysis. (a) Vascular refilling as a difference between lymphatic absorption and total transcapillary filtration; (b) transcapillary fluid filtration flows from blood to tissues through large (LP), small (SP), and ultrasmall (UP) pores; (c) mean capillary blood pressure $\left(\mathrm{P}_{\mathrm{sc}}\right)$ and interstitial hydrostatic pressure $\left(\mathrm{P}_{\mathrm{is}}\right) ;(\mathbf{d})$ interstitial-to-plasma concentration ratio for albumin and globulins; (e) plasma oncotic pressure exerted by albumin and globulins; (f) interstitial oncotic pressure exerted by albumin and globulins.

Hemodialysis. During the simulated HD procedure the transcapillary fluid filtration is subject to substantial changes (see Fig. 3a). At the beginning of the procedure, when the priming saline is infused to the virtual patient, the total filtration increases, which is due to the relatively sudden reduction in the plasma oncotic pressure exerted by albumin and globulins following hemodilution by the saline (see Fig. 3e). During HD the total transcapillary filtration from blood to tissues progressively decreases until it eventually reverses into transcapillary absorption of fluid from the interstitial space at circa $1 \mathrm{~h}$ into the dialysis session. This results mainly from the reversion of flow direction through the small pores due to increasing plasma oncotic pressure induced by dialyzer ultrafiltration combined with a decrease in capillary blood pressure-see Fig. 3c,e (note that the reversion of flow direction through small pores occurs circa $0.5 \mathrm{~h}$ earlier than that of the total transcapillary filtration). The fluid filtration across the large pores decreases to a much lower extent given that the oncotic pressure gradient across the large pores is much lower due to low reflection coefficients of albumin and globulins (see Table 1). The changes in water filtration across ultrasmall pores are opposite to those for small pores (a progressive increase), which is due to the fact that the reduced leakage of negatively charged proteins during HD entails the increased transcapillary flow of "other anions", which in the model are assumed to move according to the electroneutrality condition - this leads to the extra osmotic pressure gradient developing across ultrasmall pores, which draws more vascular water through this transport channel.

As fluid is removed from the body via ultrafiltration, the volume and the pressure of the interstitial fluid decreases (see Fig. 3c). This leads to a progressive increase in the interstitial oncotic pressure (see Fig. 3f), which partly reduces the forces driving transcapillary fluid absorption (the vascular refilling mechanism). It also leads to a slight reduction in the lymph absorption. The interstitial-to-plasma ratio of albumin remains during HD relatively stable at below 0.5 (see Fig. 3d). The analogous ratio for globulins is slightly below 0.4 . Both values are within the range reported in the literature $\left.{ }^{4,35-37}\right)$. 

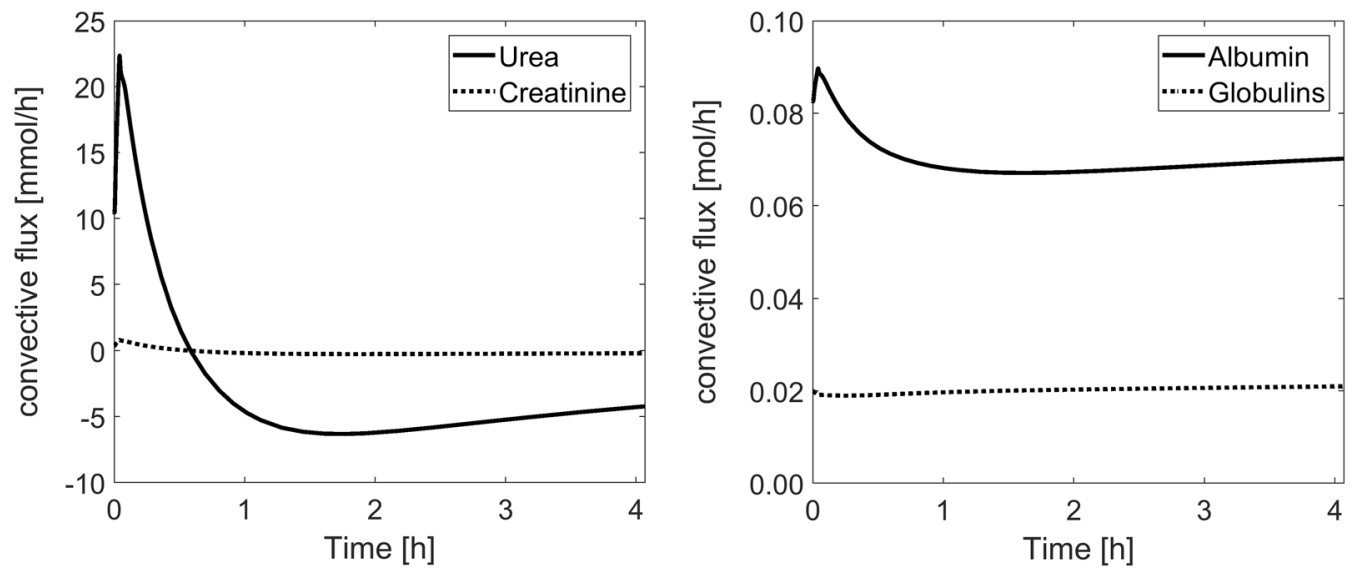

Figure 4. Model-simulated transcapillary convective fluxes of albumin, globulins, urea and creatinine during hemodialysis procedure.

Given the substantial changes in the magnitude and direction of transcapillary water flows through small pores (see Fig. 3b), the convective fluxes of individual solutes are also subject to intradialytic changes, as shown in Fig. 4. For small solutes and albumin, following the initial rise in the transcapillary convective flux (due to increased capillary filtration through small pores after the infusion of the priming saline), the transcapillary convection decreases due to decreasing filtration through small pores. For small solutes, after certain time (slightly over $0.5 \mathrm{~h}$ ), the transcapillary convective leakage of solutes reverses into transcapillary absorption and remains so until the end of dialysis. For globulins, no initial rise in the convective flux can be observed, as these proteins travel only via large pores, for which the rate of filtration is not affected by the infusion of the priming saline.

The relative changes in mean arterial blood pressure (MAP) and total blood volume (TBV) during the simulated HD (without the pre-dialysis procedure of filling the extracorporeal circuit) for different fractions of total capillary hydraulic conductivity attributed to ultrasmall pores $\left(\alpha_{U P}\right)$ or large pores $\left(\alpha_{\text {LP }}\right)$ are shown in Figs. 5 and 6.

Sensitivity analysis. In Fig. 7, we show the results of our sensitivity analysis, i.e., the relative sensitivity of the mean arterial pressure at the end of the simulated 4-h HD session with respect to model parameters related to the 3PM of the capillary wall. For clarity, we only show the parameters with the associated relative sensitivity greater than 0.01 . For all 27 studied parameters the relative sensitivities are lower than 0.04 (in absolute terms) meaning that a $10 \%$ change in the parameter value would lead to less than $0.4 \%$ change in the studied model output (MAP). Among the studied parameters, the relatively highest impact on the end-of-dialysis MAP was found for the whole-body hydraulic conductivity of capillary walls (LpS), fraction of LpS contributed by ultrasmall pores $\left(\alpha_{U P}\right)$, parameters related to albumin (radius, charge and MW), osmotic coefficient of bicarbonate ions, and charge of "other anions" (assumed -2) whose transport across the capillary wall in the model is governed by electroneutrality condition and hence is related to the transport of negatively charged proteins (mainly albumin).

Figure 8 shows the analogous relative sensitivities of the end-of-dialysis mean arterial pressure to 137 other model parameters or dialysis settings, as mentioned in the Methods section. For clarity, we only show the parameters with the associated relative sensitivity greater than 0.1 (among the parameters not shown, 99 parameters were associated with relative sensitivities lower than 0.01 ). The relatively highest impact on the end-of-dialysis MAP was found-unsurprisingly-for the ultrafiltration rate and the parameters related to the transport of sodium and chloride across the dialyzer membrane (concentrations in plasma and dialysis fluid, plasma water fraction, and the Donnan factors), and-somewhat less intuitively-for a couple of parameters related to the cardiac function (initial heart rate and the maximal stroke volume of the right ventricle).

\section{Discussion}

As far as we know, the model presented in this study is the first compartmental model of the human cardiovascular system with baroreflex regulation integrated with the model of whole-body water and solute transport that takes into account the structural (porous) properties of the capillary endothelium.

Due to substantial dialysis-induced changes in the transcapillary flow conditions through individual types of capillary pores, particularly through small and ultrasmall pores, the convective transport of solutes across the capillary walls is also subject to intradialytic changes. This is particularly important for the transcapillary transport of proteins known to occur mostly by convection ${ }^{18}$. As the magnitude and direction of flows through different types of capillary pores change during HD, the effective reflection or sieving coefficients for individual solutes also change, and hence the classic homogeneous membrane model, which assumes constant reflection coefficients, would not be able to accurately describe the aforementioned changes in the convective transport of proteins, particularly in the case of albumin, which can pass through both large and small pores.

As far as the $3 \mathrm{PM}$ is concerned, the most challenging aspect of using this model is to establish the relative number of pores of different size and to divide among them the assumed total capillary hydraulic conductivity 

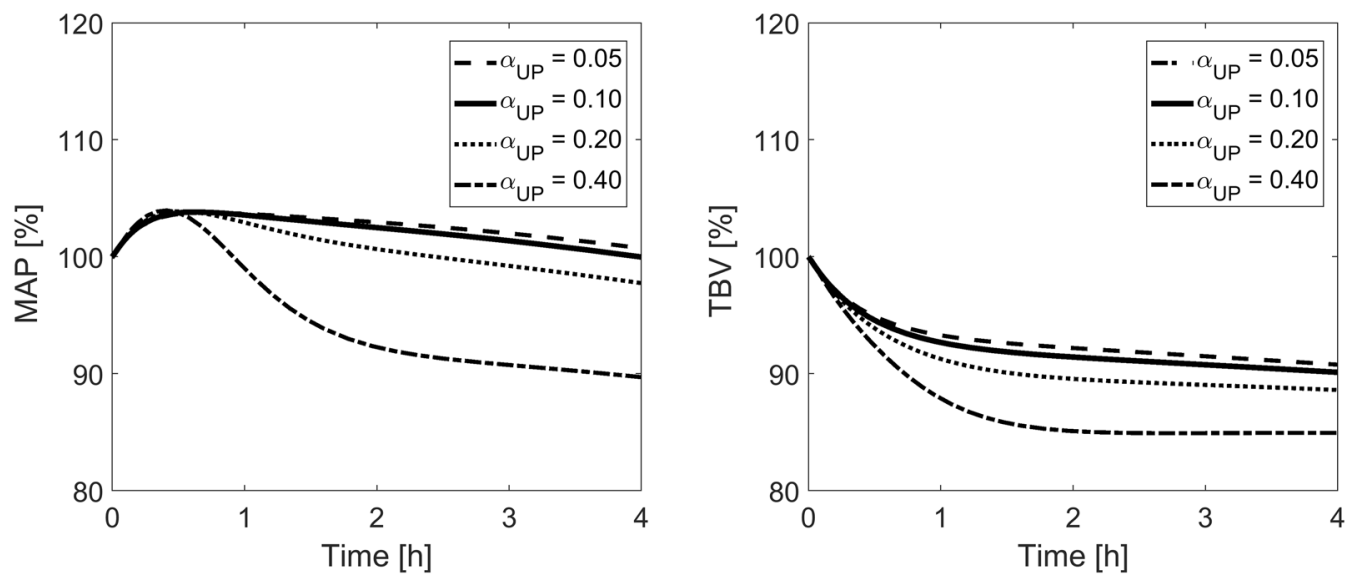

Figure 5. Model-simulated relative changes of mean arterial blood pressure (MAP) and total blood volume (TBV) during hemodialysis for different fractions of the capillary hydraulic conductivity attributed to ultrasmall pores $\left(\alpha_{U P}\right)$ with the fraction attributed to large pores kept unchanged at the basal value of 0.05 .

(LpS). This is even more challenging in the studies such as ours, where we consider transcapillary fluid and solute exchange on the whole-body level with a lumped (average) capillary wall representing capillary walls from all body tissues. It is particularly difficult to assign the hydraulic capacity of ultrasmall pores that are present only in the endothelium of continuous capillaries (assumed in this study for the whole body), thus not existing in other types of capillaries (less common on the whole-body level). Based on data by Rippe and Haraldsson ${ }^{18}$ and Michel and Curry ${ }^{38}$, in the base scenario we attributed $5 \%$ of LpS to large pores and $10 \%$ to ultrasmall pores, with the remaining $85 \%$ attributed to small pores. However, much higher values for the UP fraction in various tissues were proposed by other authors, in particular $~ 30 \%$ estimated by Kellen and Bassingthwaighte ${ }^{39}, 40 \%$ claimed by Wolf ${ }^{13}$ or $50 \%$ suggested by Pappenheimer ${ }^{40}$. According to our study, the fraction of LpS contributed by ultrasmall pores on the whole-body level $\left(\alpha_{U P}\right)$ has very little influence on the transcapillary steady-state conditions. Changing $\alpha_{U P}$ between 0.05 and 0.50 led to marginal changes in the observed transcapillary escape rate of albumin $\left(\mathrm{TER}_{\mathrm{Alb}}\right)$ or mean capillary blood pressure, despite an increase in water filtration through UP from 0.8 to $9.2 \%$ of the total filtration (see Table 4 ). On the other hand, during HD, which induces extensive systemic perturbations in terms of fluid and solute imbalances, $\alpha_{U P}$ plays a much more important role (see Fig. 5). From the results of our study, it seems that high values of $\alpha_{U P}(0.4$ or higher $)$ are rather unlikely in an otherwise healthy dialysis patient, as they lead to a significantly larger decrease in the simulated values of TBV and MAP-much greater than should be expected for the assumed (mostly normal) parameters of the cardiovascular system and the relatively moderate ultrafiltration of $2 \mathrm{~L}$. This can be also interpreted as an indication that patients with a high value of $\alpha_{U P}$ i.e. a relatively large number of ultrasmall pores on the whole-body level (e.g. due to large amount of muscle or fat tissue), may be possibly more prone to intradialytic hypotension. This hypothesis could be further investigated in the future by attempting to assess the relative amounts of tissues with continuous capillaries versus tissues with other types of capillaries in patients undergoing HD therapy. Note that the importance of ultrasmall pores in our analysis of HD results mainly from the osmotic pressure gradient exerted by "other anions", which during HD travel in the model in the opposite direction to proteins in order to keep capillary plasma and interstitial fluid electroneutral. This may be an oversimplification, as there may be more complex processes and mechanisms affecting the distribution of electrical charge across the capillary wall following a reduced leakage of negatively charged proteins. Nevertheless, several studies have shown the important role of ultrasmall pores (or aquaporins) in non-steady conditions, for instance during peritoneal dialysis ${ }^{14,41-44}$.

The results are somewhat opposite for the fraction of large pores $\left(\alpha_{\mathrm{LP}}\right)$. Changing $\alpha_{\mathrm{LP}}$ between 0.03 and 0.08 led to relatively minor changes in the hemodynamic response to HD (see Fig. 6) but provided largely different initial steady states of the modeled system with filtration through large pores changing between 32 and $61 \%$ of the total filtration (see Table 5). Looking at the steady-state values of TER $\mathrm{Alb}_{\mathrm{b}}$ and the mean capillary pressure obtained for different $\alpha_{L P}$ it seems that the value chosen for our base scenario $\left(\alpha_{L P}=0.05\right)$ is quite likely with $\mathrm{TER}_{\mathrm{Alb}} \approx 5 \% / \mathrm{h}, \mathrm{P}_{\mathrm{sc}} \approx 15.5 \mathrm{mmHg}$ and interstitial-to-plasma albumin ratio below 0.5 . A similar value for $\alpha_{\mathrm{LP}}$ was obtained in the previous study from our group ${ }^{37}$ (although that study assumed $\alpha_{\mathrm{SP}}=0.60$ ).

The importance of the parameter $\alpha_{U P}$ was confirmed in our sensitivity analysis, where it was found to be one of the parameters of the 3PM with the relatively highest influence on the end-of dialysis MAP. It should be noted that, as shown in Figs. 7 and 8, several other parameters of the model have a relatively higher impact on the endof-dialysis MAP compared to $\alpha_{U P}$; however, the values of those parameters are unlikely to differ largely from the values assumed in the model (perhaps with the exception for LpS), and hence, even if the model seems to be more sensitive to those parameters, their relatively low physiological range reduces their relative importance in the model. The value of $\alpha_{U p}$, on the other hand, carries a much higher uncertainty, as shown by the largely different values provided in the aforementioned studies (e.g. $50 \%$ vs $10 \%$ ), which justifies our model-based investigation of how the value of this parameter affects the cardiovascular response to HD. Note also that the generally low 

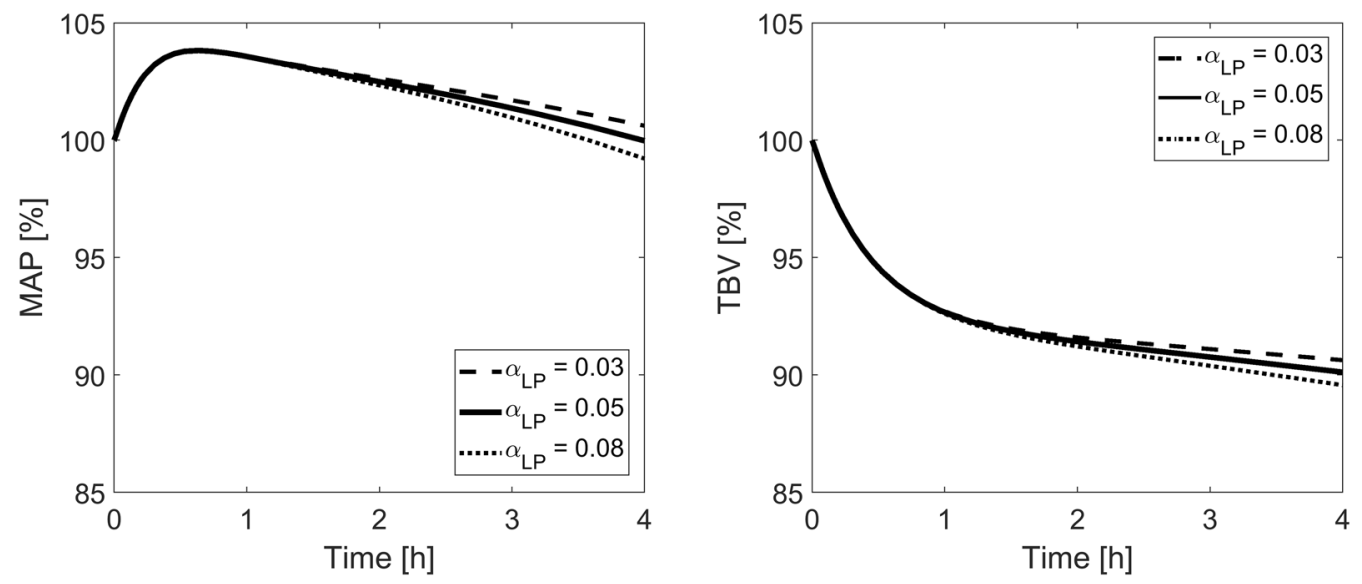

Figure 6. Model-simulated relative changes of mean arterial blood pressure (MAP) and total blood volume (TBV) during hemodialysis for different fractions of the capillary hydraulic conductivity attributed to large pores $\left(\alpha_{\mathrm{LP}}\right)$ with the fraction attributed to ultrasmall pores kept unchanged at the basal value of 0.10 .

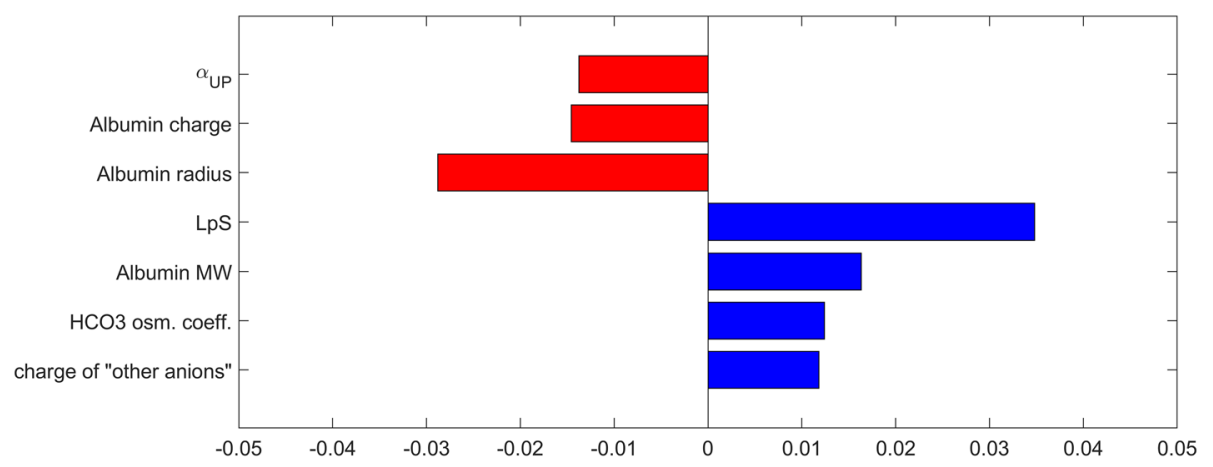

Figure 7. Relative sensitivity of the end-of-dialysis mean arterial pressure (MAP) to model parameters related to the three-pore model of the capillary wall. Only parameters with the relative sensitivity greater than 0.01 are shown.

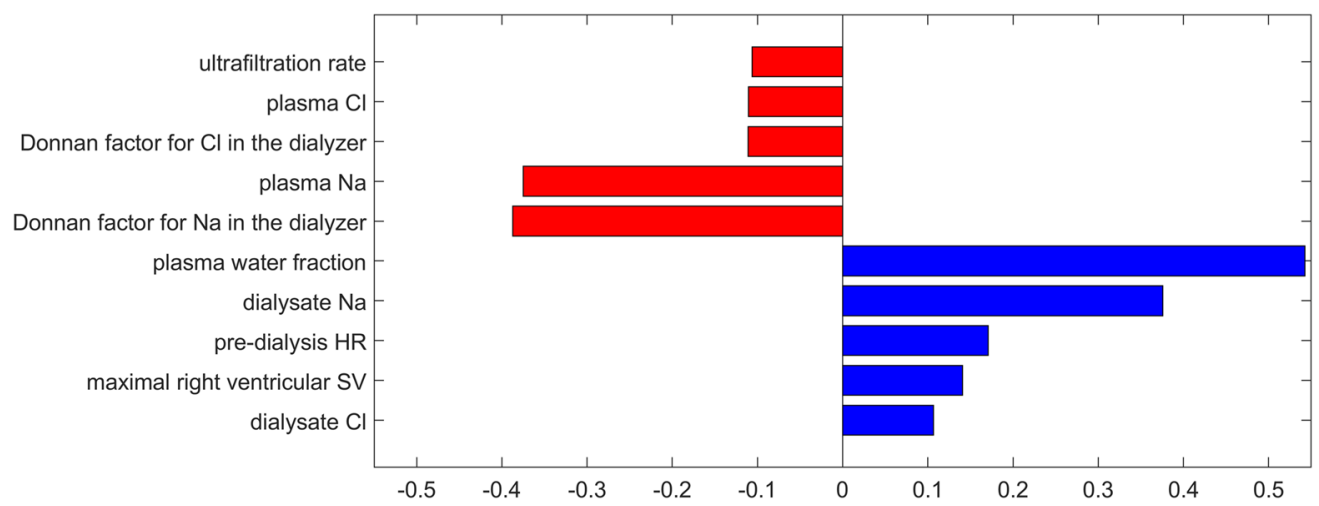

Figure 8. Relative sensitivity of the end-of-dialysis mean arterial pressure (MAP) to model parameters other than those related to the three-pore model of the capillary wall. Only parameters with the relative sensitivity greater than 0.1 are shown.

values of calculated relative sensitivities of MAP to all model parameters indicate that our model is relatively robust and resistant to isolated changes of individual parameter values.

It should be noted that patients on hemodialysis often have various cardiovascular diseases or conditions, such as hypertension or autonomic dysfunction, and hence the pre-dialysis pressures and volumes of individual 
cardiovascular compartments as well as the parameters of the baroreflex mechanisms (gains, amplitudes, and time constants) may be different than those assumed in our model for a healthy man. In consequence, the cardiovascular response to HD in such patients, in particular intradialytic changes of the mean arterial blood pressure, may differ from that presented by our simulations. Our sensitivity analysis showed that four baroreflex parameters (out of 17) have a similar or higher impact on the end-of-dialysis MAP compared to $\alpha_{U P}$ (amplitude of the peripheral resistance regulation and gains of the mechanisms controlling peripheral resistance, venous unstressed volume and heart contractility). Therefore, substantial changes in the values of these baroreflex parameters (e.g. a substantial reduction of the gain or amplitude of peripheral resistance regulation) may have a similar effect on dialysis-induced changes in MAP as that suggested for a high fraction of ultrasmall pores.

In this study, we assumed a pre-dialysis plasma sodium level of $138 \mathrm{mmol} / \mathrm{L}$ and a common dialysate sodium concentration of $140 \mathrm{mmol} / \mathrm{L}^{31,32}$. With these values, taking into account the corrections for plasma water fraction and the Gibbs-Donnan effect, there will be a slight diffusion of sodium from the dialysis fluid to the patient. With the use of a higher dialysate sodium (e.g. in patients with hyponatremia or intradialytic hypotensive symptoms, such as muscle cramps), the intradialytic sodium diffusion would be accordingly higher, which, as shown by the sensitivity analysis, would obviously impact the intradialytic changes of blood pressure. However, given the relatively high rates of transcapillary diffusion of small solutes, such as sodium, and relatively small intradialytic changes in plasma sodium (compared, for instance, to changes in plasma urea concentration), this would not affect our conclusions with regard to the relative impact of the fraction of ultrasmall pores.

Our study has, however, certain limitations. Firstly, as already mentioned, we analyze the transcapillary transport of water and solutes on the whole-body level assuming that the properties of the capillary walls are the same across the entire body (including pulmonary capillaries) and equivalent to those of continuous capillaries found in skeletal muscles, thus we do not take into account the fact that different organs and tissues may have different microvascular properties and hence different rates of local transcapillary fluid and solute exchange during HD. Similarly, in our study, the lymph absorption from the interstitial space reflects the whole-body lymph flow, including the pulmonary lymph. However, the properties of the pulmonary capillary walls and the pulmonary interstitial fluid differ from those of other tissues ${ }^{45}$. Secondly, we assume that all parameters describing the transcapillary transport processes are the same for both sides of the capillary wall (e.g. the same solute reflection coefficients regardless of flow direction) and that they are constant. During HD, however, the properties of the capillary walls may potentially change, thus influencing the analyzed transport processes. Moreover, we ignore the recent theory on the protein gradient developing between the bulk interstitial fluid and the fluid beneath the so-called glycocalyx ${ }^{3,46}$, according to which, when calculating the Starling forces across the capillary wall, one should use the (lower) oncotic pressure of the subglycocalyx fluid and not that of the bulk interstitial fluid $^{2,3}$. Finally, our model of baroreflex regulation is also subject to some limitations which we discuss in our earlier work ${ }^{17}$.

In summary, our study showed that during non-steady conditions, such as $\mathrm{HD}$, the 3PM reveals complex transcapillary transport phenomena, which are not possible to be captured by a standard homogeneous membrane model with fixed parameter values. We also showed that the fraction of hydraulic conductivity attributed to ultrasmall pores may play an important role in the transcapillary water transport during HD and the cardiovascular response to $\mathrm{HD}$. It seems hence, that the $3 \mathrm{PM}$ - with a particular attention to the ultrasmall pore fraction-should be the preferred conceptual model for detailed investigations of vascular refilling mechanisms, the rate of capillary protein leakage, and the maintenance of cardiovascular stability during HD.

\section{Data availability}

The detailed description of the mathematical model used in this study as well as the values of all model parameters and initial conditions may be found in our previous work, as mentioned in the manuscript.

Received: 15 July 2020; Accepted: 15 October 2020

Published online: 30 October 2020

\section{References}

1. Grände, P. O., Holbeck, S. \& Persson, J. In Perioperative Fluid Therapy (eds Hahn, R. G. et al.) (CRC Press, New York, 2007)

2. Levick, J. R. Capillary filtration-absorption balance reconsidered in light of dynamic extravascular factors. Exp. Physiol. 76(6), 825-857 (1991).

3. Levick, J. R. \& Michel, C. C. Microvascular fluid exchange and the revised Starling principle. Cardiovasc. Res. 87, 198-210 (2010).

4. Schneditz, D. et al. Nature and rate of vascular refilling during hemodialysis and ultrafiltration. Kidney Int. 42(6), 1425-1433 (1992).

5. Pietribiasi, M. et al. Kinetics of plasma refilling during hemodialysis sessions with different initial fluid status. ASAIO J. 61(3), 350-356 (2015).

6. Pietribiasi, M., Wojcik-Zaluska, A., Zaluska, W. \& Waniewski, J. Does the plasma refilling coefficient change during hemodialysis sessions?. Int. J. Artif. Organs 41(11), 706-713 (2018).

7. Mitsides, N., Pietribiasi, M., Waniewski, J., Brenchley, P. \& Mitra, S. Transcapillary refilling rate and its determinants during haemodialysis with standard and high ultrafiltration rates. Am. J. Nephrol. 50(2), 133-143 (2019).

8. Davenport, A. Intradialytic complications during hemodialysis. Hemodial. Int. 10(2), 162-167 (2006).

9. Reeves, P. B. \& McCausland, F. R. Mechanisms, clinical implications, and treatment of intradialytic hypotension. Clin. J. Am. Soc. Nephrol. 13, 12141017 (2018).

10. Waniewski, J. Theoretical Foundations for Modelling of Membrane Transport in Medicine and Biomedical Engineering (Institute of Computer Science, Polish Academy of Sciences, Warsaw, 2015).

11. Waniewski, J. Mathematical modeling of fluid and solute transport in hemodialysis and peritoneal dialysis. J. Membrane Sci. 274, 24-37 (2006).

12. Wolf, M. B. Identification of microvascular transport pathways in skeletal muscle. Am. J. Physiol. Heart Circ. Physiol. 267, H383H399 (1994). 
13. Wolf, M. B. A three-pathway pore model describes extensive transport data from mammalian microvascular beds and frog microvessels. Microcirculation 9, 497-511 (2002).

14. Rippe, B., Rosengren, B. I. \& Venturoli, D. The peritoneal microcirculation in peritoneal dialysis. Microcirculation 8, 303-320 (2001).

15. Pstras, L., Thomaseth, K., Waniewski, J., Balzani, I. \& Bellavere, F. Mathematical modelling of cardiovascular response to the Valsalva manoeuvre. Math. Med. Biol. 34(2), 261-292 (2017).

16. Pstras, L., Thomaseth, K., Waniewski, J., Balzani, I. \& Bellavere, F. Modeling pathological hemodynamic responses to the valsalva maneuver. J. Biomech. Eng. 139, 6 (2017).

17. Pstras, L. \& Waniewski, J. Mathematical Modelling of Haemodialysis: Cardiovascular Response, Body Fluid Shifts, and Solute Kinetics (Springer Nature Switzerland AG, Cham, 2019).

18. Rippe, B. \& Haraldsson, B. Transport of macromolecules across microvascular walls: The two-pore theory. Physiol. Rev. 74(1), $163-219$ (1994).

19. Yashiro, M., Hamada, Y., Matsushima, H. \& Muso, E. Estimation of filtration coefficients and circulating plasma volume by continuously monitoring hematocrit during hemodialysis. Blood Purif. 20(6), 569-576 (2002).

20. Feher, J. Quantitative Human Physiology. An introduction 182-198 (Academic Press, San Diego, 2017).

21. Choi, J. H., Lee, H. J. \& Moon, S. H. Effects of electrolytes on the transport phenomena in a cation-exchange membrane. J. Colloid Interface Sci. 238, 188-195 (2001).

22. Kiriukhin, M. Y. \& Collins, K. D. Dynamic hydration numbers for biologically important ions. Biophys. Chem. 99, 155-168 (2002).

23. Lefebvre, X., Palmeri, J. \& David, P. Nanofiltration theory: An analytic approach for single salts. J. Phys. Chem. B 108, 16811-16824 (2004).

24. Hussain, A. A., Abashar, M. E. \& Al-Mutaz, I. S. Effect of ion sizes on separation characteristics of nanofiltration membrane systems. J. King Saud Univ 19, 1-19 (2006).

25. Kadhim, M. J. \& Gamaj, M. I. Estimation of the diffusion coefficient and hydrodynamic radius (stokes radius) for inorganic ions in solution depending on molar conductivity as electro-analytical technique-a review. J. Chem. Rev. 2(3), 182-188 (2020).

26. Sciancalepore, A. G. et al. A bioartificial renal tubule device embedding human renal stem/progenitor cells. PLoS One 9(1), e87496 (2014).

27. Sharma, A. C. et al. A general photonic crystal sensing motif: Creatinine in bodily fluids. J. Am. Chem. Soc. 126, 2971-2977 (2004).

28. Armstrong, J. K., Wenby, R. B., Meiselman, H. J. \& Fisher, T. C. The hydrodynamic radii of macromolecules and their effect on red blood cell aggregation. Biophys. J. 87, 4259-4270 (2004).

29. Rashid, R. et al. Novel use for polyvinylpyrrolidone as a macromolecular crowder for enhanced extracellular matrix deposition and cell proliferation. Tissue Eng. 20(12), 994-1002 (2014).

30. Feher, J. Quantitative Human Physiology. An Introduction (Elsevier, Amsterdam, 2012).

31. Flythe, J. E. \& McCausland, F. R. Dialysate sodium: Rationale for evolution over time. Semin. Dial. 30(2), 99-111 (2017).

32. Movilli, E. et al. Role of dialysis sodium gradient on intradialytic hypertension: An observational study. Am. J. Nephrol. 38(5), 413-419 (2013).

33. RodríguezFernández, M., Banga, J. R. \& Doyle, F. J. III. Novel global sensitivity analysis methodology accounting for the crucial role of the distribution of input parameters: Application to systems biology models. Int. J. Robust Nonlinear Control 22(10), 1082-1102 (2012).

34. Qian, G. \& Mahdi, A. Sensitivity analysis methods in the biomedical sciences. Math. Biosci. 323, 108306 (2020).

35. Aukland, K. \& Reed, R. K. Interstitial-lymphatic mechanisms in the control of extracellular fluid volume. Physiol. Rev. 73(1), 1-78 (1993).

36. Ellmerer, M. et al. Measurement of interstitial albumin in human skeletal muscle and adipose tissue by open-flow microperfusion. Am. J. Physiol. Endocrinol. Metab. 278(2), E353-356 (2000).

37. Pietribiasi, M., Waniewski, J., Załuska, A., Załuska, W. \& Lindholm, B. Modelling transcapillary transport of fluid and proteins in hemodialysis patients. PLoS One 11(8), e0159748 (2016).

38. Michel, C. C. \& Curry, F. E. Microvascular permeability. Physiol. Rev. 79(3), 703-761 (1999).

39. Kellen, M. R. \& Bassingthwaighte, J. B. Transient transcapillary exchange of water driven by osmotic forces in the heart. Am. J. Physiol. Heart Circ. Physiol. 285, H1317-H1331 (2003).

40. Pappenheimer, J. R. In Capillary Permeability (eds Crone, C. \& Vassen, N. A.) (Munksgaord, Copenhagen, 1970).

41. Devuyst, O. \& Rippe, B. Water transport across the peritoneal membrane. Kidney Int. 85, 750-758 (2014).

42. Stachowska-Piętka, J., Waniewski, J., Vonesh, E. \& Lindholm, B. Changes in free water fraction and aquaporin function with dwell time during continuous ambulatory peritoneal dialysis. Artif. Organs 34, 1138-1143 (2010).

43. Ni, J. et al. Aquaporin-1 plays an essential role in water permeability and ultrafiltration during peritoneal dialysis. Kidney Int. 69, $1518-1525$ (2006)

44. Stachowska-Pietka, J., Poleszczuk, J., Flessner, M. F., Lindholm, B. \& Waniewski, J. Alterations of peritoneal transport characteristics in dialysis patients with ultrafiltration failure: Tissue and capillary components. Nephrol. Dial. Transplant 34(5), 864-870 (2019).

45. Guyton, A. C. \& Hall, J. E. Textbook of Medical Physiology 11th edn. (Elsevier Saunders, Philadelphia, 2006).

46. Adamson, R. H. et al. Oncotic pressures opposing filtration across non-fenestrated rat microvessels. J. Physiol. 557, 889-908 (2004).

\section{Acknowledgements}

The core mathematical model used in this paper was developed as part of L.P.s doctoral research project 'Mathematical modelling of cardiovascular response to haemodialysis' funded by the Polish National Science Centre (agreement no. UMO-2016/20/T/ST7/00289).

\section{Author contributions}

L.P. conceived and designed the study, developed the computational model and performed simulation experiments; L.P and J.W. analyzed and interpreted the results; L.P. drafted the manuscript and prepared the figures; J.W. and B.L. reviewed, revised and edited the manuscript; all authors approved the final version of the manuscript.

\section{Competing interests}

B.L. is employed by Baxter Healthcare Corporation. L.P. and J.W. have no competing interests.

\section{Additional information}

Supplementary information is available for this paper at https://doi.org/10.1038/s41598-020-75687-1.

Correspondence and requests for materials should be addressed to L.P.

Reprints and permissions information is available at www.nature.com/reprints. 
Publisher's note Springer Nature remains neutral with regard to jurisdictional claims in published maps and institutional affiliations.

(c) (i) Open Access This article is licensed under a Creative Commons Attribution 4.0 International License, which permits use, sharing, adaptation, distribution and reproduction in any medium or format, as long as you give appropriate credit to the original author(s) and the source, provide a link to the Creative Commons licence, and indicate if changes were made. The images or other third party material in this article are included in the article's Creative Commons licence, unless indicated otherwise in a credit line to the material. If material is not included in the article's Creative Commons licence and your intended use is not permitted by statutory regulation or exceeds the permitted use, you will need to obtain permission directly from the copyright holder. To view a copy of this licence, visit http://creativecommons.org/licenses/by/4.0/.

(c) The Author(s) 2020 